\title{
People with gambling disorder and risky alcohol habits benefit more from motivational interviewing than from cognitive behavioral group therapy
}

Henrik Josephson, Per Carlbring, Lars Forsberg, Ingvar Rosendahl

Background. Effective psychological treatment, including cognitive behavioral therapy and motivational interviewing (MI), is available for people with problematic gambling behaviors. To advance the development of treatment for gambling disorder, it is critical to further investigate how comorbidity impacts different types of treatments. The purpose of this study was to investigate whether screening for risky alcohol habits can provide guidance on whether people with gambling disorder should be recommended cognitive behavioral group therapy (CBGT) or MI. Methods. The present study is a secondary analysis of a previous randomized controlled trial that compared the effects of CBGT, MI and a waitlist control group in the treatment of disordered gambling. Assessment and treatment was conducted at an outpatient dependency clinic in Stockholm, Sweden, where 53 trial participants with gambling disorder began treatment. A modified version of the National Opinion Research Centre DSM-IV Screen for gambling problems was used to assess gambling disorder. The Alcohol Use Disorders Identification Test (AUDIT) was used to screen for risky alcohol habits. Results. The interaction between treatment and alcohol habits was significant and suggests that patients with gambling disorder and risky alcohol habits were better helped by MI, while those without risky alcohol habits were better helped by CBGT. Conclusions. The results support a screening procedure including the AUDIT prior to starting treatment for gambling disorder because the result of the screening can provide guidance in the choice of treatment. Patients with gambling disorder and risky alcohol habits are likely to be best helped if they are referred to MI, while those without risky alcohol habits are likely to be best helped if they are referred to CBGT. 
1 People with gambling disorder and risky alcohol habits benefit more from motivational

2 interviewing than from cognitive behavioral group therapy

3 Henrik Josephson ${ }^{1}$, Per Carlbring ${ }^{2}$, Lars Forsberg ${ }^{1}$ \& Ingvar Rosendahl ${ }^{1}$

$4{ }^{1}$ Department of Clinical Neuroscience, Karolinska Institute, Stockholm, Sweden

5 2Department of Psychology, Stockholm University, Stockholm, Sweden

6

7 Corresponding author:

8 Henrik Josephson ${ }^{1}$

9 Centre for Psychiatry Research

10 Department of Clinical Neuroscience

11 Karolinska Institute \& Stockholm Health Care Services, Stockholm County Council

12 Liljeholmstorget 7B, SE-117 63 Stockholm, Sweden

13 Phone: +46727222354

14 E-mail: henrik.josephson@ki.se 


\section{Introduction}

Worldwide, .3\% to $5.3 \%$ of adults suffer from gambling problems (Wardle et al., 2010).

Disordered gambling is a diagnosis described in the Diagnostic and Statistical Manual of Mental Disorders, 5th edition (DSM-5; American Psychiatric Association, 2013), as a persistent and recurrent problematic gambling behavior leading to clinically significant impairment or distress. The diagnosis shares several characteristics with substance-related disorders. Common features include preoccupation, increased tolerance, loss of control, withdrawal symptoms, and family and job disruption (American Psychiatric Association, 2013).

Meta-analyses and systematic reviews have provided evidence for the efficacy of psychological treatment for gambling disorder (Gooding \&Tarrier, 2009; Hodgins, Stea \& Grant, 2011; Yakovenko et al., 2015). Results from a meta-analysis revealed that various forms of cognitive behavioral therapy (CBT) and motivational interviewing (MI) showed large and significant effect sizes in the 0-3-months period post treatment, with enduring effects at the 24-month (or later) follow-up (Gooding \&Tarrier, 2009). Effect sizes were highly significant despite variability in the populations being treated, severity of gambling problem, and type of gambling (Gooding \&Tarrier, 2009).

It is well known that gambling disorder is highly comorbid with other psychiatric disorders (Bischof et al., 2013; Lorains, Cowlishaw \& Thomas, 2011; Petry, Stinson \& Grant, 2005). Data derived from a large national sample in the United States indicate that the most frequently reported lifetime comorbid condition among people with gambling disorder was alcohol use disorder (73.2\%; Petry, Stinson \& Grant, 2005); the corresponding figure in a large German 
study was $61.7 \%$ (Bischof et al., 2013). What is even more interesting from a clinical perspective is that the results of a recent review on co-morbidity among gamblers seeking treatment for their gambling problems point in the same direction, with rates of current alcohol use disorders at 21.2\% (Dowling et al., 2015). Gamblers' alcohol consumption while gambling and the effect of alcohol on their gambling behavior are of particular interest. Most regular video lottery terminal (VLT) gamblers (73\%) said that they prefer to drink alcoholic beverages while gambling (Stewart et al., 2002), and up to $80 \%$ of gamblers without a gambling disorder diagnosis reported a consumption of four to ten alcoholic drinks during their last gambling session (Baron \& Dickerson, 1999). In other words, gamblers often drink while gambling, and it has been shown that those who do tend to engage in more risky gambling behaviors (Cronce \& Corbin, 2010; Ledgerwood et al., 2009). People with gambling disorder and co-occurring alcohol use disorders reported greater levels of problematic gambling (Welte et al., 2004) and were more likely to have psychiatric comorbidity than those without alcohol use disorders (Abdollahnejad, Delfabbro \& Denson, 2014). Gamblers with alcohol problems are also at an increased risk of relapse after quitting gambling (Hodgins \& El-Guebaly, 2010).

In a study that mapped the drinking patterns of people with gambling disorder, entry into gambling treatment was temporally associated with reduction in alcohol use, but gamblers with risky alcohol habits were still less likely to adhere to gambling treatment (Rash, Weinstock \& Petry, 2011). One study suggested that alcohol problems were linked to poor compliance in individual CBT treatment for gambling disorder (Milton et al., 2002). The study reported that people with gambling disorder comorbid with alcohol problems were 2.5 times more likely to drop out of treatment than people with gambling disorder without alcohol problems (Milton et 
61 al., 2002). This result has failed to be replicated in subsequent research on individual CBT

62 (Leblond, Ladouceur \& Blaszczynski, 2003) and multimodal CBT (Stinchfield, Kushner \&

63 Winters, 2005). Reports on the relationship between alcohol problems and dropout in the

64 treatment of gambling disorder are of clinical interest, but there is no research on how co-morbid

65 conditions such as alcohol problems affect the outcome of patients who actually pursue and

66 remain in treatment for gambling disorder. Neither is there any research on whether different

67 treatment forms, such as CBT and MI, differ in sensitivity to co-occurring alcohol problems. To

68 advance the development of treatment for gambling disorder, it is critical to investigate further

69 how comorbidity impacts different types of treatments for gambling disorder (Dowling et al.,

70 2015; Hodgins, Stea \& Grant, 2011; Petry, Stinson \& Grant, 2005).

Because an alcohol use disorder is the most common comorbid condition among people with gambling disorder (Bischof et al., 2013 Dowling et al., 2015; Petry, Stinson \& Grant, 2005) and contributes to a loss of control over gambling (Cronce \& Corbin, 2010; Ledgerwood et al., 2009.), more severe gambling problems (Welte et al. 2004), higher rates of psychiatric comorbidity (Abdollahnejad, Delfabbro \& Denson, 2014), impaired adherence to gambling treatment (Milton et al., 2002; Rash, Weinstock \& Petry, 2011), and an increased likelihood of gambling (Hodgins \& El-Guebaly, 2010), it is of great clinical interest to see whether the condition has different affects on the outcome of widely used therapies, such as cognitive behavioral group therapy (CBGT) and MI. The purpose of this study was to investigate whether screening for risky alcohol habits can provide guidance on whether people with gambling disorder should be recommended CBGT or MI. The analysis in the present study is based on a data set from a previous randomized controlled trial that compared the effects of CBGT, MI, and 
84 a waitlist control in the treatment of gambling disorder (Carlbring et al., 2010). At baseline the

85 three randomized groups had no significant differences in gambling-related measures, levels of

86 depression, or alcohol consumption. In the trial the CBGT and MI groups showed greater

87 reductions in the symptoms of gambling disorder than the no-treatment control group. Both

88 CBGT and MI generated significant within-group decreases on gambling-related outcome

89 measures up to the 12-month follow-up. However, no differences in outcome measures were

90 found between CBGT and MI at any point (Carlbring et al., 2010).

91

92

\section{Methods}

93

\section{Design}

94 The present study is a secondary analysis of a previous randomized controlled trial that compared the effects of CBGT, MI, and a waitlist control in the treatment of gambling disorder (Carlbring et al., 2010). The study was approved by the regional ethics committee in Stockholm 97 (2005/5:5), and informed written consent was collected from each participant.

\section{Recruitment and Participants}

Between June 2005 and December 2006, 80 people with gambling disorder began treatment at an outpatient dependency clinic in Stockholm, Sweden. A total of 53 trial participants were included in the present study. Reasons for exclusion were not providing baseline data $(n=2)$ and not providing data at the 6-month treatment follow-up $(n=25)$. Participants received two cinema tickets for participating in the treatment follow-up.

\section{Diagnostic and data collection procedures}


107 Prior to starting treatment, all participants went through a 60- to 90-minute in-person interview 108 conducted by a clinical psychologist trained in the assessment procedures. The interview 109 included demographic questions and a set of self-report measures, including the National 110 Opinion Research Centre DSM-IV Screen for gambling problems (NODS; Gerstein et al., 1999), 111 the Timeline Followback (TLFB) calendar (Weinstock, Whelan \& Meyers, 2004) and the

112 Alcohol Use Disorders Identification Test (AUDIT; Babor et al., 2001). The participants were 113 asked to fill out the set of self-report measures again at the 6-month follow-up.

\section{Measures}

The NODS (Gerstein et al., 1999), modified to assess gambling at one month instead of one year, was used to assess gambling disorder. The use of the 1-month version of the instrument has not seemed to affect the instrument's reliability or validity. A comparison of the internal consistency between the NODS lifetime, past-year. and 3-month versions has shown Cronbach's alphas of $.86, .87$, and .87 respectively (Wulfert et al., 2005). The total score, ranging from 0 to 10 , is normally used to identify pathological gambling (scores 5 and above) according to DSM-IV

122 (American Psychiatric Association, 2000). The instrument was modified to assess gambling disorder according to DSM-5 by eliminating the illegal acts criterion and lowering the threshold for diagnosis to 4 criteria of a possible 9 . Recent research indicates that the increased sensitivity of the DSM-5 gambling disorder diagnosis successfully identifies a broader group of gamblers with clinically significant gambling-related problems (Rennert et al., 2014). Participants included

127 in the present study were those assigned with NODS scores of 4 through 9 at baseline. A TLFB 128 calendar (Weinstock, Whelan \& Meyers, 2004) was used to assess the number of days gambled 129 in the last 30 days. 
131 The AUDIT (Babor et al., 2001) was used to assess risky alcohol habits. The instrument is a 10-

132 item multiple-choice self-report inventory with a total score ranging from 0 to 40 . Scores of $0-7$

133 for men and 0-5 for women (Zone 1) indicate low-risk drinking. Scores of 8-15 for men and 6-

13413 for women (Zone 2) indicate hazardous and harmful alcohol use. Scores of 16-19 for men

135 and 14-17 for women (Zone III) indicate a medium level of alcohol problems with a probable

136 alcohol-related diagnosis. Finally, scores above 19 for men and 17 for women (Zone IV) indicate

137 a high level of alcohol problems, with a probable alcohol-related diagnosis. The AUDIT

138 accurately assesses the severity of problematic alcohol use behaviors across a wide range of

139 contexts and populations at risk (Allen et al., 1997). When administered as part of a larger

140 battery of tests in a primary care setting, the AUDIT showed test-retest reliability after a 6-week

141 interval with a correlation of $r=.88$ and an internal consistency reliability of $\alpha=.85$ (Daeppen et

142 al., 2000). In the present study AUDIT scores were analyzed in two ways: first with Zones II-IV

143 as three separate categories vs. Zone I (reference) and then as a dichotomized factor with Zones

144 II-IV combined vs. Zone I.

\section{Treatments}

147 The CBGT treatment $(n=25)$ was administered in closed groups conducted as one 3-hour

148 session per week for 8 weeks. The treatment was manualized (Ortiz, 2006) and each session

149 focused on a set theme. The sessions included psychoeducation, exercises, and distribution and

150 follow-up of homework. A recurrent feature throughout the treatment was exercises aimed at

151 reducing the urge to gamble through imaginary exposure and response prevention. The treatment

152 was focused partly on cognitive restructuring and partly on encouraging clients to try alternative 
153 behavioral strategies. Another important treatment component dealt with identifying personal

154 high-risk situations for gambling and increasing participants' skills to cope with these situations 155 in a more functional way.

156

157 The MI treatment $(n=28)$ was administered individually in four 50-minute sessions. The first 158 two sessions were one week apart, and the last two sessions followed at three-week intervals, for 159 a total treatment time of 8 weeks - the same as the CBGT condition. The therapists used the MI 160 approach as described by Miller and Rollick in 2002, including showing empathy, eliciting the 161 participant's own reasons for making a change, collaborating with and supporting the participant 162 in autonomy, developing the discrepancy between ongoing problematic behaviors and the 163 participant's internal goals and values, and supporting the participant's confidence in their own 164 abilities. Techniques such as open-ended questions and reflective listening were used throughout 165 the sessions. If the patients were ready to change, they were encouraged to make a decision about 166 changing their gambling behavior and to make a change plan. The therapists had access to a 167 semi-structured manual in which these standard MI principles were described and exemplified in 168 the context of problem gambling (Forsberg, Forsberg \& Knifström, 2010).

\section{Treatment fidelity}

171 The therapists administering the CBGT received continuous supervision. All sessions were 172 audio-taped and 20\% were randomly selected for coding by an independent licensed clinical 173 psychologist with psychotherapist training and experience in the specific treatment method.

174 According to the treatment manual (Ortiz, 2006) a total of 375 agenda points should be covered.

175 The coding showed $93 \%$ adherence to the manual. 
177 To test MI treatment integrity, all sessions were audio-taped and $20 \%$ of the sessions were

178 randomly selected to be coded by independent and blinded coders using the Motivational

179 Interviewing Treatment Integrity Code 2.0 (MITI; Moyers et al., 2003). The MI competency in

180 the delivered sessions was deemed acceptable using the given reference values for MI

181 proficiency in the coding manual (Moyers et al., 2003). Supervision of the MI treatment was

182 accomplished through assessment of the therapists' audio-taped sessions. Results from the

183 coding were used to facilitate specific feedback.

\section{Statistical analyses}

186

187

Analyses were conducted using SPSS 22.0 and STATA 14.0. Independent $t$-tests and Chi-square tests were used to determine whether the two treatment groups differed in pre-treatment characteristics. The same test statistics were also used to investigate whether participants who were lost at follow-up $(n=25)$ differed in pre-treatment characteristics from participants who completed the follow-up measurements. Analysis of covariance (ANCOVA) of the NODS scores and number of gambling days in the last 30 days at the 6-month follow-up was used, with the NODS scores and number of gambling days in the last 30 days at pre-treatment used as the models' quantitative control variables. The final two models (one for NODS scores and the other for number of gambling days) had two factors, treatment (MI vs. CBGT) and AUDIT (risky vs. not risky alcohol habits), with an interaction term included in the models. Marginal means were calculated from the ANCOVA model and visualized (for the NODS scores) via a bar-plot of the margins. To assess the difference in NODS scores between the CBGT and the MI treatment adjusted for alcohol habits, contrasts of discrete marginal effects were estimated and tested. 


\section{Results}

201

202

203

204

205

206

207

208

209

210

211

212

213

214

215

216

217

218

219

220

221

\section{Pre-treatment variables}

Table 1 shows baseline point estimates and the distribution of some basic characteristics of the participants $(n=53)$. No statistically significant differences in characteristics were found between the two treatment groups.

Insert Table 1 about here

\section{NODS scores at 6-month follow-up}

The interaction between treatment and alcohol habits in the ANCOVA-model was significant [F $(1,48)=5.39 ; p=.025]$, and suggests that the effect of treatment depends on the patient's alcohol habits. Although none of the factors in Table 1 differed significantly between treatment groups, we adjusted the model for gender, age, minority status, income level, gambling debts, and treatment attendance and found that none of these variables markedly changed the main estimates. Therefore, only the unadjusted estimates are presented. Marginal means calculated from the ANCOVA model showed that patients with gambling disorder and risky alcohol habits who received MI treatment had a mean NODS score of 1.9 at the 6-month follow-up. As the low average NODS score suggests, a strikingly large proportion (81.8\%) of the participants in this group no longer met the criteria for gambling disorder at the 6-month follow-up. For patients with gambling disorder and risky alcohol habits who received CBGT, the corresponding NODS score was 4.0 , with a lower proportion of participants $(30.0 \%)$ who no longer met the criteria for 
222 gambling disorder at follow-up. The contrasts between MI and CBGT, shown in Figure 1, were

223 significantly different between participants with no risky alcohol habits and participants with

224 risky alcohol habits $[\mathrm{t}(48)=2.32 ; p=.025]$.

225

226

227

228

229

230

231

232

233

234

235

236

237

238

239

240

241

242

243

244

\section{Insert Figure 1 about here}

To confirm the results for the NODS scores, we used the same ANCOVA model with the

number of gambling days per month as outcome measure. Unfortunately, there were only 39 observations for this outcome measure compared with 53 observations for the NODS scores, which might explain why no results from these analyses became significant. However, among participants in Zone I (alcohol habits), the means and standard deviations were 11.34 (1.87) for the MI treated and 8.86 (2.73) for those treated by CBGT. Among participants in Zones II-IV, the mean and standard deviations were 5.14 (3.45) for the MI treated and $11.18(2.57)$ for those treated by CBGT. In other words, the results for number of gambling days were in line with the result for the NODS scores. These results suggest a better treatment outcome for CBGT than for MI among participants in Zone I and a better treatment outcome for MI than for CBGT among participants in Zones II-IV. In the first analysis that was performed, the AUDIT scores were analyzed with Zones II-IV as three separate categories compared with Zone I (reference). The risk estimate for Zone III versus Zone I was higher than the risk estimate for Zone II versus Zone I. 


\section{Analyses of missing data}

246 There were 25 patients who did not participate in the 6-month follow-up, equally distributed

247 between the two treatment groups, CBGT $(n=13)$ and MI $(n=12)$. There were no statistically

248 significant differences (no $p$-values lower than .40) in terms of sex, age, or pretreatment scores

249 on NODS and AUDIT between those who participated in the 6-month follow-up and those who

250 did not.

251

\section{Discussion}

253 The findings in this study suggest that patients with gambling disorder respond differently to

254 CBGT and MI depending on whether or not they have risky alcohol habits at pre-treatment.

255 Patients with gambling disorder who also have risky alcohol habits appear to have a better

256 chance of benefitting from MI, and patients with no risky alcohol habits appear to have a better

257 chance of benefitting from CBGT. The results are clinically relevant because they can be used to

258 facilitate the referral of patients with gambling disorder to the treatment that will help them the

259 best. These findings raise the question of why MI appears to be more efficient than CBGT in

260 treating patients with gambling disorder and risky alcohol habits, and why CBGT appears to be

261 more efficient when patients do not have risky alcohol habits.

262

263 In a recent study on the personality traits of people with gambling problems with and without

264 alcohol dependence, individuals with gambling disorder and co-occurring lifetime alcohol

265 dependence reported a personality style characterized by resistance to externally motivated

266 treatment approaches (Lister, Milosevic \& Ledgerwood, 2015). In MI, patient behaviors

267 characterized by resistance have been a focus of treatment, and such resistant behavior might be 
268 better addressed by MI than CBGT treatment. MI is a non-authoritarian, collaborative method

269 that focuses on building intrinsic motivation (Miller \& Rollick, 2013). The individually

270 administered MI also offers more opportunities to tailor treatment to patient needs. MI is a

271 compassionate treatment during which the patient is likely to feel comfortable raising personal

272 issues (Miller \& Rollick, 2013) that may pose obstacles to treatment if they are not given space.

273 Risky alcohol habits could be addressed in MI treatment if it would help the patient to move

274 towards the change goal, to stop or reduce gambling. The advantage of being able to address

275 multiple behavior targets in MI treatment may have had a significant impact on the outcome

276 because the two addictive behaviors are likely to trigger, reinforce, and maintain each other.

277 Alcohol is usually readily available at casinos, racetracks. and other gambling environments, and

278 gambling under the influence of alcohol is associated with higher risk-taking (Cronce \& Corbin,

279 2010; Ledgerwood et al., 2009). Conversely, events that occur during gambling (e.g., winning

280 and losing) may trigger alcohol consumption (Zack et al., 2005). Multiple behavior targets in MI

281 treatment have been studied in other fields of addiction, and have proven to be effective in

282 motivating people to simultaneously reduce their usage of tobacco, alcohol, and cannabis

283 (McCambridge \& Strang, 2004). In a review on smoking cessation during substance abuse

284 treatment, Baca and Yahne (2009) concluded that targeting smoking cessation enhances outcome

285 success and reduces substance use.

286

287 An additional advantage of the MI treatment over CGBT is that risky alcohol habits might have

288 the same origins as the gambling disorder (Stewart et al., 2008). The MI therapist is therefore

289 able to address risky alcohol habits in the treatment of gambling disorder, and the reasons for 
290 both alcohol consumption and gambling could then be highlighted and tackled from different 291 angles.

292

293 In the CBGT treatment, on the other hand, the possibility of tailoring treatment to fit any 294 comorbid conditions is very small because the treatment is in a group format and strictly follows 295 a manual (Ortiz, 2006). The superior effect of CBGT on patients who did not have risky alcohol 296 habits can probably be explained by the fact that CBGT was an extensive treatment that included 297 a wide range of psychoeducative elements, exercises, and homework assignments that all 298 addressed various aspects of problem gambling (Ortiz, 2006)

299

\section{Strengths and limitations of the study}

The major strength of this study is that it addresses the important issue of moderators of

302

303

304

305

306

307

308

309

310

311

312 treatment effects. It highlights a factor that is highly correlated to gambling disorder and appears to moderate the outcome of treatment. The two treatment arms compared were evidence-based effective treatment methods for gambling problems, and treatment outcome was measured six months post-treatment, which implies that the results were persistent. The potential moderator (risky alcohol habits) included in the analysis was selected for two main reasons. First, it is the most common comorbid condition among people with gambling disorder (Bischof et al., 2013; Petry, Stinson \& Grant, 2005); second, earlier findings indicate that the condition is an aggravating factor in treatment that correlates with impaired adherence to treatment (Milton et al., 2002; Rash, Weinstock \& Petry, 2011), and increased risk of gambling relapse (Hodgins \& El-Guebaly, 2010). 
313 A limitation of this study is the small sample size, which made it difficult to include additional

314 potential moderating variables in the model that would have been interesting to analyze.

315 Additional potentially predictive comorbid conditions, such as drug use, mood, anxiety, and

316 personality disorders should be included in future research. Unfortunately, a large number of

317 patients dropped out at follow-up and were excluded from the analysis. This makes it necessary

318 to be cautious in interpreting the results. The modified version of the NODS (assessing gambling

319 at one month instead of one year) has not been evaluated. However, shortening the window of

320 time from one year to 3 months does not appear to affect the instrument's reliability or validity

321 (Wulfert et al., 2005). Moreover, an apparent benefit of a shorter-term version of the NODS is

322 that it can serve as a convenient treatment outcome measure. Another limitation is that it is

323 unclear to what extent these results can be explained by different modes of treatment (individual

324 vs. group) and to what extent they can be explained by unique factors inherent in each treatment.

325 A final limitation is that there was no control group. It is therefore unknown whether the

326 participants' reported reductions of symptoms of gambling disorder during the 6-month post

327 treatment period were the results of the treatment or spontaneous recovery. About one third of

328 individuals with gambling problems are believed to recover without formal treatment (Slutske,

329 2006). However, we have no reason to believe that the rate of spontaneous recovery should be

330 different between treatment groups.

\section{Generalizability}

334 There were missing data at the 6-month follow-up. However, there were no statistically

335 significant differences between those who participated and those who did not participate in the 
336 follow-up in terms of sex, age, severity of problem gambling, and alcohol problems at baseline.

337 It appears reasonable to conclude that it is possible to generalize the findings to gamblers seeking

338 treatment for gambling problems serious enough to meet the criteria for gambling disorder. The

339 findings are interesting from a health-planning perspective, and are valid for both CBGT and MI,

340 which are two commonly used evidence-based treatments for gambling disorder (Gooding \&

341 Tarrier, 2009; Hodgins, Stea \& Grant, 2011; Yakovenko et al., 2015)

342

\section{Future research}

344 First, the results from this study need to be replicated to ensure that these associations are not

345 sample-specific. In order to confirm our results, future studies should state a priori the hypothesis

346 that people with gambling disorder and risky alcohol habits will benefit more from MI than from

347 CBGT, and that people with gambling disorder but no risky alcohol habits will be helped more

348 by CBGT than by MI. Further research would improve the validity of the findings if an

349 intention-to-treat analysis were conducted. Moreover, further research is needed to investigate

350 how other comorbid conditions, such as depression and anxiety, affect the efficacy of treatment.

351 It would also be useful to learn more about the impact of comorbidity on individual CBT.

\section{Conclusions}

354 The results support a screening procedure including the AUDIT prior to the start of treatment for 355 gambling disorder because the result of the screening can provide guidance in the choice of 356 treatment. Patients with gambling disorder and risky alcohol habits are more likely to be helped

357 if they are referred to MI treatment, while those without risky alcohol habits are likely to be best 358 helped if they are referred to CBGT. 
360 Acknowledgments

361 The authors would like to thank Sarah Heinrich for her valuable comments made to a previous

362 version of this manuscript.

363

364 References

365 Abdollahnejad R, Delfabbro P \& Denson L. 2014. Psychiatric co-morbidity in problem and 366 pathological gamblers: Investigating the confounding influence of alcohol use disorder. 367 Addictive Behaviors 39: 566 - 572.

Allen JP, Litten RZ, Fertig JB, Babor T. 1997. A Review of Research on the Alcohol Use

Disorders Identification Test (AUDIT). Alcohol Clin Exp Res 21: 613 - 619.

American Psychiatric Association. 2013. Diagnostic and statistical manual of mental disorders.

5th edition. Washington, DC: American Psychiatric Association.

374

375

Babor TF, Higgins-Biddle JC, Saunders JB, Monteiro MG. 2001. AUDIT The Alcohol Use

Disorders Identification Test: Guidelines for use in Primary Care 2nd ed. World Health

Organization.

Baca CT \& Yahne CE. 2009. Smoking cessation during substance abuse treatment: What you need to know. Journal of Substance Abuse Treatment 36: 205 - 219. 
382 Baron E \& Dickerson M. 1999. Alcohol consumption and self-control of gambling behavior.

383 Journal of Gambling Studies 15: 3 - 15.

384

385 Bischof A, Meyer C, Bischof G, Kastirke N, John U \& Rumpf HJ. 2013. Comorbid axis I

386 disorders among subjects with pathological, problem, or at risk gambling recruited from the

387 general population in Germany: Results of the PAGE study. Psychiatry Research 210: 1065 $388 \quad 1070$.

389

390 Carlbring P, Jonsson J, Josephson H \& Forsberg L. 2010. Motivational interviewing versus

391 cognitive behavioural group therapy in the treatment of problem and pathological gambling: A

392 randomized controlled trial. Cognitive Behavior Therapy 39: 92 - 103.

393

394 Cronce JM \& Corbin WR. 2010. Effects of alcohol and initial gambling outcomes on within395 session gambling behavior. Experimental and Clinical Psychopharmacology 18: 145 - 157.

Daeppen JB, Yersin B, Landry U, Pécoud A, Decrey H. 2000. Reliability and Validity of the Alcohol Use Disorders Identification Test (AUDIT) Imbedded Within a General Health Risk

Screening Questionnaire: Results of a Survey in 332 Primary Care Patients. Alcoholism Clinical and Experimental Research 24: 659 - 665.

401

402

Dowling NA, Cowlishaw S, Jackson AC, Merkouris SS, Francis KL, Christensen DR. 2015.

Prevalence of psychiatric co-morbidity in treatment-seeking problem gamblers: A systematic review and meta-analysis. Australian \& new Zealand Journal of Psychiatry 49: 519-539. 
406 Forsberg L, Forsberg K \& Knifström E. 2010. Motiverande behandling vid spelproblem och 407 spelberoende (Motivational Interviewing for pathological gambling). Östersund: Statens 408 folkhälsoinstitut.

409

410 Gerstein D, Murphy S, Toce M, Hoffaman J, Palmer A, Johnson R, Larison C, Chuchro L, Buie 411 T, Engelman L, Hill MA. 1999. Gambling impact and behavior study: Report to the national 412 gambling impact study commission. Chicago: National Opinion Research Center.

414 Gooding P, Tarrier N. 2009. A systematic review and meta-analysis of cognitive-behavioural 415 interventions to reduce problem gambling: Hedging our bets? Behav Res Ther 47:592-607. 416

417 Hodgins DC \& el-Guebaly N. 2010. The influence of substance dependence and mood disorders 418 on outcome from pathological gambling: Five-year follow-up. Journal of Gambling Studies 26: $419117-127$.

420

421 Hodgins DC, Stea JN, Grant JE. 2011. Gambling disorders. Lancet 378: 1874 - 1884.

423 Leblond J, Ladouceur R, Blaszczynski A. 2003. Which pathological gamblers will complete 424 treatment? British Journal of Clinical Psychology 42: 205-209.

425

426 Ledgerwood DM, Alessi SM, Phoenix N \& Petry N. 2009. Behavioral assessment of impulsivity 427 in pathological gamblers with and without substance use disorder histories versus healthy 
428 controls. Drug and Alcohol Dependence 105: 89 - 96.

429

430 Lister JL, Milosevic A \& Ledgerwood DM. 2015. Personality traits of problem gamblers with 431 and without alcohol dependence. Addictive Behaviors 47: 48 - 54.

432

433

Lorains FK, Cowlishaw S \& Thomas SA. 2011. Prevalence of comorbid disorders in problem 434 and pathological gambling: systematic review and meta-analysis of population surveys.

435 Addiction 106: 490 - 498.

436

437

McCambridge J \& Strang J. 2004. The efficacy of single session motivational interviewing in 438 reducing drug consumption and perceptions of drug-related risk and harm among young people: 439 results from a multi-site cluster randomized trial. Addiction 99: 39 - 52

440

441

Miller WR \& Rollnick S. 2002. Motivational Interviewing: Preparing People for Change, $2^{\text {nd }}$ 442 edition: New York: Guilford Press.

443

444

Miller WR \& Rollick S. 2013. Motivational Interviewing: Helping people change, $3^{\text {rd }}$ edition:

445 New York: Guilford Press.

446

447 Milton S, Crino R, Hunt C, Prosser E. 2002. The effect of compliance-improving interventions

448 on the cognitive-behavioural treatment of pathological gambling. Journal of Gambling Studies

449 18: $207-229$.

450 
451 Moyers T, Martin T, Manual J \& Miller WR. 2003. The Motivational Interviewing treatment

452 Integrity Code. Albuquerque: University of New Mexico.

453

454 Ortiz L. 2006. Till spelfriheten! Kognitiv beteendeterapi vid spelberoende (Cognitive behavioral 455 therapy for pathological gambling) Stockholm: Natur \& Kultur.

456

457 Petry NM, Stinson FS, Grant BF. 2005. Comorbidity of DSM-IV pathological gambling and 458 other psychiatric disorders: Results from the National Epidemiologic Survey on Alcohol and 459 Related Conditions. J Clin Psychiatry 66:564 - 574.

460

461 Rash CJ, Weinstock J \& Petry NM. 2011. Drinking patterns of pathological gamblers before, 462 during, and after gambling treatment. Psychology of Addictive Behaviors 25: 664 - 674.

463

464 Rennert L, Denis C, Peer K, Gelernter J, Lynch K G, Kranzler H R. 2014. DSM-5 Gambling 465 disorder: Prevalence and characteristics in a substance use disorder sample. Experimental and 466 clinical psychopharmacology 22: 50 - 56.

467

468 Slutske WS. 2006. Natural recovery and treatment-seeking in pathological gambling: Results of 469 two U.S. national surveys. American journal of psychiatry 163: $297-302$.

471 Stewart SH, McWilliams LA, Blackburn JR \& Klein RM. 2002. A laboratory-based

472 Investigation of relations among video lottery terminal (VLT) play, negative mood, and alcohol 
473 consumption in regular VLT players. Addictive Behaviors 27: 819 - 835.

474

475 Stewart SH, Zack M, Collins P, Klein RM \& Fragopoulos F. 2008. Subtyping pathological

476 gamblers on the basis of affective motivations for gambling: Relations to gambling problems,

477 drinking problems, and affective motivations for drinking. Psychology of Addictive Behaviors

$478 \quad 22: 257-268$.

479

480 Stinchfield R, Kushner MG, Winters KC. 2005. Alcohol use and prior substance abuse treatment

481 in relation to gambling problem severity and gambling treatment outcomes. Journal of Gambling

482 Studies 21: $273-297$.

483

484 Wardle H, Moody A, Spence S, Orford J, Volberg R, Jotangia D, Griffiths M, Hussey D, Dobbie 485 F. 2011. British Gambling Prevalence Survey 2010. London: National Centre for Social 486 Research.

487

488 Welte JW, Barnes GM, Wieczorek WF, Tidwell MCO \& Parker JC. 2004. Risk factors for 489 pathological gambling. Addictive Behaviors 29: 323 - 335.

490

491 Weinstock J, Whelan J, Meyers A. Behavioral Assessment of Gambling: An application of the 492 Timeline Followback Method. Psychological Assessment 2004; Vol 16:(1) 72-80.

493

494 Wulfert E, Hartley J, Lee M, Wang N, Franco C \& Sodano R. 2005. Gambling Screens: Does 
495 Shortening the Time Frame Affect their Psychometric Properties? Journal of Gambling Studies $496 \quad 21: 521-536$.

497

498 Yakovenko I, Quigley L, Hemmelgarn BR, Hodgins DC \& Ronksley P. 2015. The efficacy of 499 motivational interviewing for disordered gambling: Systematic review and meta-analysis.

500 Addictive Behaviors 43: 72-82.

501 Zack M, Stewart SH, Klein RM, Loba P \& Fragopoulos F. 2005. Contingent gambling-drinking 502 patterns and problem drinking severity moderate implicit gambling-alcohol associations in 503 problem gamblers. Journal of Gambling Studies 21: 325 - 354. 
Table 1. Participants' characteristics at pre-treatment including 95\% confidence interval (CI95\%)

\begin{tabular}{|c|c|c|c|c|c|}
\hline \multirow[b]{2}{*}{ Characteristics } & \multicolumn{2}{|c|}{$\operatorname{CBGT}(n=25)$} & \multicolumn{2}{|c|}{ MI $(n=28)$} & \multirow[t]{2}{*}{$p$-value } \\
\hline & Mean & CI95\% & Mean & CI95\% & \\
\hline NODS No risky alcohol & 6.1 & $5.1-7.2$ & 6.0 & $5.1-6.9$ & .84 \\
\hline habits & 6.2 & $5.3-7.1$ & 5.7 & $5.1-6.3$ & .33 \\
\hline \multicolumn{6}{|l|}{ NODS Risky alcohol habits } \\
\hline AUDIT No risky alcohol habits & 2.2 & $.9-3.5$ & 3.6 & $2.3-4.9$ & .11 \\
\hline AUDIT Risky alcohol habits & 15.7 & $12.3-19.1$ & 16.1 & $8.8-23.4$ & .92 \\
\hline AUDIT-C & 4.1 & $2.7-5.5$ & 4.1 & $2.9-5.2$ & .99 \\
\hline BDI & 25.8 & $20.0-31.7$ & 25.6 & $20.7-30.5$ & .95 \\
\hline BAI & 18.8 & $13.4-24.2$ & 18.0 & $14.0-21.9$ & .81 \\
\hline Age & 43.0 & $37.5-48.4$ & 40.8 & $35.9-45.6$ & .53 \\
\hline Gambling debt, 1000 & 10.2 & $5.2-15.3$ & 8.7 & $4.0-13.4$ & .65 \\
\hline & Proportion & & Proportion & & $p$-value \\
\hline Female & 20.0 & & 17.9 & & .84 \\
\hline Prior gambling treatment & 40.0 & & 46.4 & & .64 \\
\hline Prior psychiatric treatment & 44.0 & & 57.1 & & .34 \\
\hline Only elementary school & 32.0 & & 32.1 & & .99 \\
\hline Immigrant & 24.0 & & 39.3 & & .23 \\
\hline Unemployed & 16.0 & & 14.3 & & .86 \\
\hline Low income & 16.0 & & 21.4 & & .61 \\
\hline \multicolumn{6}{|l|}{ Primary gambling on: } \\
\hline Video lottery terminals & 56.0 & & 46.4 & & .49 \\
\hline Horse/sport betting & 16.0 & & 25.0 & & .42 \\
\hline Casino/poker & 12.0 & & 10.7 & & .88 \\
\hline Other & 16.0 & & 17.9 & & .86 \\
\hline
\end{tabular}




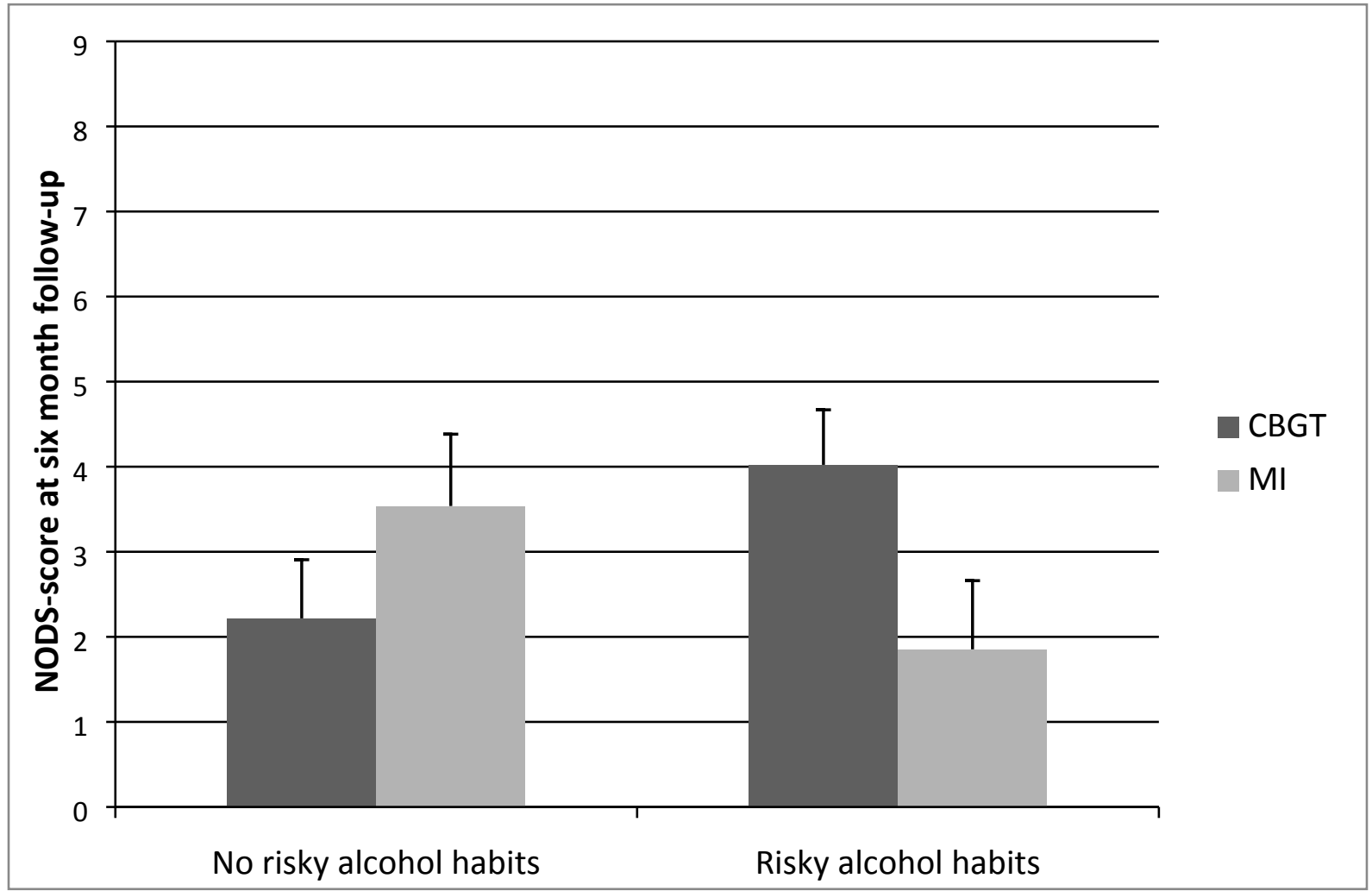

Figure 1. Marginal means and standard errors for interaction effects between treatment and alcohol habits 